\title{
ETFRC: Enhanced TFRC for Media Traffic
}

\author{
Mohammad A. Talaat \\ M. Sc. Eng. \\ University of Menoufia \\ Menoufia, Egypt
}

\author{
Magdi A. Koutb \\ Prof. Dr. Eng. \\ University of Menoufia \\ Menoufia, Egypt
}

\author{
Hoda S. Sorour \\ Dr. Eng. \\ University of Menoufia \\ Menoufia, Egypt
}

\begin{abstract}
The remarkable increase in media traffic over Internet is expected to worsen its congestion state. TCP-friendly rate control protocol TFRC is one of the most promising congestion control techniques developed so far. TFRC has been thoroughly tested in terms of being TCP-friendly, responsive, and fair. Yet, its impact on the visual quality and the peak signal-to- noise ratio PSNR of the media traffic traversing Internet is still questionable. In this paper we aimed to point out the enhancement required for TFRC that enables producing the maximum PSNR value for Internet media traffic. Firstly, we suspected the default value of $\mathrm{Nfb}$ that represents the frequency of feedback messages sent by TFRC receiver to its sender every round-trip time RTT to be the optimum for our goal. Secondly, we managed to modify the mechanism of TFRC by changing this default value of $\mathrm{Nfb}$ over a range of values aiming to reach the required optimum. Thirdly, we investigated the effect of such variation over a simulated network environment to study its effect on the resulting PSNR for a number of arbitrary video sequences. Finally, our simulations results showed that running TFRC with $\mathrm{Nfb}=4$ led to reaching the maximum PSNR values among all the examined values of $\mathrm{Nfb}$ including its default value. We hereby suggest using an enhanced TFRC that we abbreviated as ETFRC which has the $\mathrm{Nfb}$ value set to four as a replacement for the traditional TFRC to enable reaching higher PSNR for media traffic over Internet.
\end{abstract}

\section{General Terms}

Congestion Control

\section{Keywords}

TFRC, PSNR.

\section{INTRODUCTION}

The amount of media traffic traversing Internet has remarkably increased over the last decade. Applications pushing media traffic such as video on demand VoD, video conferencing, and various video streaming websites have been lately invading the cyber space. The best-effort existing IP infrastructure was not primarily designed to suffice the quality of service QoS requirements of such traffic. Both of the current UDP and TCP have drawbacks when used as the transport protocol for media traffic. TCP seems to break the delay constraints due to its acknowledgments; meanwhile UDP shows aggressiveness in acquiring the available bandwidth to accomplish the streaming task. UDP leaves an unfair share for the co-existing TCP flows which leads to congestion status.

During the periods of congestion; routers tend to discard legitimate packets traversing a certain bottleneck to be able to serve the aggressive media packets. Efforts have been made by researchers to control congestion; they tried to balance between allowing for QoS achievement and acting in a TCP-friendly manner at the same time.

This was via building congestion control protocols that leave a fair share of bandwidth for the concurrent TCP flows traversing across the same bottleneck. Protocols designed for this purpose have been tested regarding compatibility with the TCPfriendliness concept defined in [1]

TFRC presented in [2] was one of the congestion control protocols that managed to achieve remarkable smoothness in the variations of its rate of transmission in addition to fulfilling the TCP-friendliness conditions. For this reason TFRC was the best current promising candidate for media streaming applications, where its smoothness helps in reducing the undesired jitter of the perceived video. TFRC has been extensively tested in terms of fairness, aggressiveness, and responsiveness as in [3] and in terms of user-perceived media quality on analytical basis in [4]. Testing the visual quality of the media traffic running over TFRC in terms of its produced PSNR was made in our previous work in [5]

This paper aims to reach an enhanced version of TFRC named as ETFRC that manages to produce higher PSNR values than for media traffic over Internet. To achieve this goal a network simulation topology was built to stream a variety of arbitrary video sequences over TFRC. The Nfb parameter that represents the frequency of feedback messages per RTT was switched among different values aiming to determine its optimum value for the best visual quality of video sequences transmitted over TFRC. This quality is measured in terms of the produced RSNR values for the streamed videos. The video sequences files used in our simulations varied in their content types, frame lengths, and motion complexity scales. Therefore, these files are a good measure for the TFRC testing purpose. The optimal value for $\mathrm{Nfb}$ was found to be "four" where the maximum PSNR values were observed; hence TFRC is suggested to enhance its mechanism to be ETFRC that uses $\mathrm{Nfb}=4$ instead of the default value used in traditional TFRC which is $\mathrm{Nfb}=1$.

The rest of this paper is organized as follows: Section II covers the TFRC literature and its modified versions tackling the media streaming task over the last decade. Section III explains the enhancement we proposed to TFRC mechanism to produce the proposed ETFRC for media traffic over Internet. Section IV explains our simulation environment, the tool-set used in simulations, the topology of the simulated network, the link parameters set, and the characteristics of the running video sequences. Section IV presents the simulations output focusing on the PSNR values for the tested TFRC in order to show that the enhancement introduced to reach our proposed ETFRC 
managed to produce the maximum PSNR values. Finally Section V presents the conclusion of this work.

\section{THE TFRC PROTOCOL}

\subsection{TCP-friendly Congestion Control}

TCP friendly congestion control schemes lies into two main categories according to [1]: (i) single rate schemes and (ii) multi-rate schemes. Unicast applications tend to utilize the single rate protocols where all recipients receive data with the same rate. This feature limits the scalability of the protocol towards bandwidth variations that exist in the path to some recipients. Multi-rate protocols are more flexible and enable the allocation of different rates for different recipients which makes it more appropriate for the multicast applications.

Each of those two top categories can be sub-divided into other two sub-categories as follows: (i) rate-based schemes and (ii) window-based schemes.

Some of the rate-based schemes apply the additive increase multiplicative decrease AIMD approach embedded in TCP. Other rate-based just tune their sending rate in accordance with a TCP model. In both cases the reliability feature of TCP is absent. Example protocols that lie in this category are RAP [6], LDA+ [7], and TFRC.

TCP itself is a window-based protocol, yet some problems should be considered when applying this mechanism on multicast connections. Multicast TCP MTCP [8] is an example protocol of this multicast category that managed to deal with these problems.

\subsection{TFRC Protocol}

TFRC is the evolution of TFRCP [9]. It was mainly developed for unicast communications but it can be adapted for multicast. Its sending rate is tuned according to the TCP complex equation (1).

$$
T=\min \left(\frac{W_{m} \cdot S}{R T T}, \frac{S}{R T T \sqrt{\frac{2 b P}{3}}+R T O \min \left(1,3 \sqrt{\frac{3 b p}{8}}\right) P\left(1+32 p^{2}\right)}\right) .
$$

Where the parameters are as follows:

- $\quad \mathrm{T}$ : TCP throughput

- $\quad$ RTT: Round-trip time

- RTO: Retransmission time-out value

- $\quad$ S: Segment size

- P: Rate of packet loss

- $\quad$ b: Number of packets acknowledged by each ACK

- $W_{m}$ : Maximum congestion window size cwnd.

TFRC uses its sophisticated mechanisms to gather the equation parameters. The average loss interval is the chosen method to fulfill the requirements of the loss rate estimation. The loss rate is measured via loss intervals through tracking the number of packets between consecutive loss events. The average of a specified number of loss intervals is calculated using decaying weights so that old loss intervals contribution in this average is less. The loss rate is considered as the inverse of the average loss interval size.

Some additional mechanisms are adopted to prevent TFRC from responding aggressively to single loss events, and to guarantee that the sending rate adapts quickly to the long intervals that are loss-free. RTT is measured by sending feedback time stamps to sender.

TFRC goes through a slow-start phase directly after starting just like TCP in order to increase its rate to reach a fair share of bandwidth. The slow-start phase is ended by reporting a loss event. TFRC receiver updates the equation parameters and feeds them back to sender to adjust its rate every round-trip time RTT. Hence a feedback message is sent once per RTT which means that $\mathrm{Nfb}=1$, leading to the recalculation of the sending rate for TFRC only once per RTT.

TFRC adopts additional delay-based congestion avoidance by adjusting the inter-packet gap which would be applied in some environments that do not support the TCP complex equation.

TFRC main advantage is its stable and smooth sending rate variations. This feature fits in the media application transmission besides being responsive to the co-existing traffic in a TCPfriendly manner.

\subsection{TFRC Enhancement Attempts}

Several attempts were made to enhance the performance of TFRC in order to suit the media traffic requirements such as what discussed in the following lines:

In [10] authors observed some performance degradation for TFRC over wireless networks, and hence they tried to customize it through a more advanced equation. This equation was reached via modeling wireless TCP rather than wired TCP. Applying this equation led to a remarkable throughput increase with about $30 \%$ over wireless networks with loss of $10 \%$ while maintaining the TFRC main features of TCP-friendliness and smoothness.

In [11] an attempt was made to enhance TFRC performance over mobile and pervasive networks that focused on overcoming data losses due to the frequent loss of connectivity. The method used was applying a mechanism that resembles Freeze-TCP when a disconnection incident is expected. Additionally, a probing mechanism to enable speedy adaptation to new network conditions was proposed.

Another enhancement was made in [12] where authors tackled the problem of keeping fairness and smoothness of TFRC media streams when existing among other streams. They proposed MulTFRC that was successful in keeping low delay values to satisfy the media QoS requirements.

In [13] the enhancement made was that authors computed the rate gap between the ideal TCP throughput and the smoothed TFRC throughput replacing it. Any rate gain from this gap was opportunistically exploited via video encoding. A frame complexity measure is specified to determine the additional rate to be used from this rate gap, and then the target rate for the encoder and the final sending rate are negotiated through the same frame of complexity. 
In [14] authors suggested incorporating the selective retransmission concept into TFRC. Retransmission of lost packets is done selectively when no congestion case is present. Selective retransmission was shown to have a significant positive effect on the streamed media quality over TFRC.

In [15] authors claimed that TFRC does not perform satisfactorily on multi-hop ad-hoc wireless networks. They saw that TFRC sending rate can be deceived by MAC layer contention effects such as retransmission and exponential backoff. Hence, they proposed enhancing TFRC by introducing RETFRC. RE-TFRC used measurements of the current round-trip time and a model of wireless delay to prevent TFRC from overloading the MAC layer while keeping its TCP-friendliness feature.

In [16] a performance analysis of a QoS-aware congestion control mechanism named guaranteed TFRC (gTFRC) was presented. gTFRC was embedded into the enhancement transport protocol (ETP) that enables protocol mechanisms to be dynamically controlled. gTFRC managed to reach a minimum guaranteed transfer rate for any given round-trip values and any network provisioning conditions as well

In [17] an extension for TFRC was suggested in order to support variable packet size streams. Variable packet size is already utilized over Internet in both video and voice over IP VoIP transmission. This enhancement of TFRC was made through a modified concept of TCP-friendliness and was validated to perform better than the original TFRC with the packets of variable sizes.

In [18] authors proposed an enhanced TFRC based on step explicit congestion notification ECN making. They found that TFRC has poor performance over wireless networks because it accounts for wireless losses as congestion. They managed to reach higher throughput via utilizing the appropriate loss differentiation and congestion notification. Their proposed enhanced TFRC kept reasonable friendliness to the co-existing TCP flows according to their simulation results.

The above enhancement attempts focused on achieving a better quality of data transmitted over TFRC while maintaining its main advantageous characteristics of being TCP- friendly and fair in acquiring a bandwidth share.

\section{ENHANCED TFRC ETFRC}

The main enhancement that we introduced to TFRC to produce ETFRC was increasing the feedback frequency represented by $\mathrm{Nfb}$ parameter from one to four. Our goal is to demonstrate through simulations that this increase had a positive feedback on the observed PSNR of the streamed video. For the sake of completeness, other effects of such increase on the TFRC mechanism of work should be discussed. In [19] authors discussed the impact of increasing the feedback frequency from different perspectives that we summarize here.

\subsection{The Impact of Increasing Nfb on TFRC}

To have a better view for the background image of TFRC, we have to understand that there are two key parameters that drive the TFRC mechanism in Eq.1 which are the loss rate $p$ and the experienced RTT. In our work we used the value of $\mathrm{Nfb}=1$ as a reference value as this frequency is considered as the default of TFRC. Simulations made in [19] showed that feedback frequencies greater than one per RTT lead to lower drop rate. It was also clear that the feedback frequency is not correlated to $p$, where both values were randomly distributed. Hence, $\mathrm{Nfb}$ has no critical effect on $\mathrm{p}$ from the macroscopic perspective. The analysis made in [19] for TFRC demonstrated the impact of increasing the feedback frequency on RTT, it showed that during the slow-start of TFRC the link suffers from underutilization as well as slow convergence. For the first hundred seconds the value of RTT in TFRC equals to that of the link propagation delay, meanwhile after this period, TFRC enters the steady state where the increase in feedback frequencies causes the experienced RTT to increase

\subsection{Increasing $\mathrm{Nfb}$ and the Sending Rate}

Researches also noted that when the feedback frequency increases the rate of the senders tend to decrease, which seems to contradict with what was expected of decrease in network congestion and the decrease in RTT consequently. The higher feedback frequency was found to improve the accuracy of the estimated RTT and the accuracy of the estimated $p$ in consequent. This enables TFRC to acquire the network resources with the minimum required sending rate due to the fact that the more accurate the sense of the network congestion parameter the faster the adaptation of TFRC to the network conditions.

\subsection{Nfb and the Network Parameters}

Increasing the feedback frequency of TFRC from one to four had a positive impact on its responsiveness. However the resulting fairness depended on how lost packets were distributed among flows. The link utilization also followed the same behavior of increase like that of responsiveness when having multiple feedbacks per RTT.

\section{SIMULATION ENVIRONMENT}

This section describes our simulation environment used to perform the PSNR evaluation of the media traffic over TFRC. The main three components of these simulations are the tool-set used, the topology of the simulated network created using ns2.30 [20], and the group of video sequences arbitrarily chosen for this purpose.

\subsection{Evalvid Toolset and Evalvid-RA}

In [21] Chih-heng Ke et al. proposed a novel and complete toolset for evaluating the quality of MPEG video delivery over simulated networks environment. This tool-set is based on the EvalVid framework [22]. They managed to let ns-2 as a general network simulator replace the EvalVid simple error simulation model through extending its connecting interfaces. This allowed researchers and practitioners in general to simulate and analyze the performance of real video streams with consideration for the video semantics under a vast range of network scenarios. The tool-set valuable feature is that it allows for the examination of the relationship between two well-known objective metrics for QoS assessment of video quality of delivery which are the PSNR and the fraction of decodable frames. As shown in Fig. 1 the concept of this tool-set is built upon creating trace files from an encoded raw video. These files are text files and are fed into the simulation environment to be used as traffic generators based on the encoded video parameters. The 
output of the simulation process can be decoded as well to produce the output video file of the simulation. The quality of the output file and the original video file can then be compared to obtain a representative PSNR value for the media quality of the produced video from simulation.
EvalVid-RA proposed in [23] is an extension of EvalVid toolset that supports the rate adaptive media content as well as the TFRC protocol in the simulation environment, thus it was chosen to be used in this work.

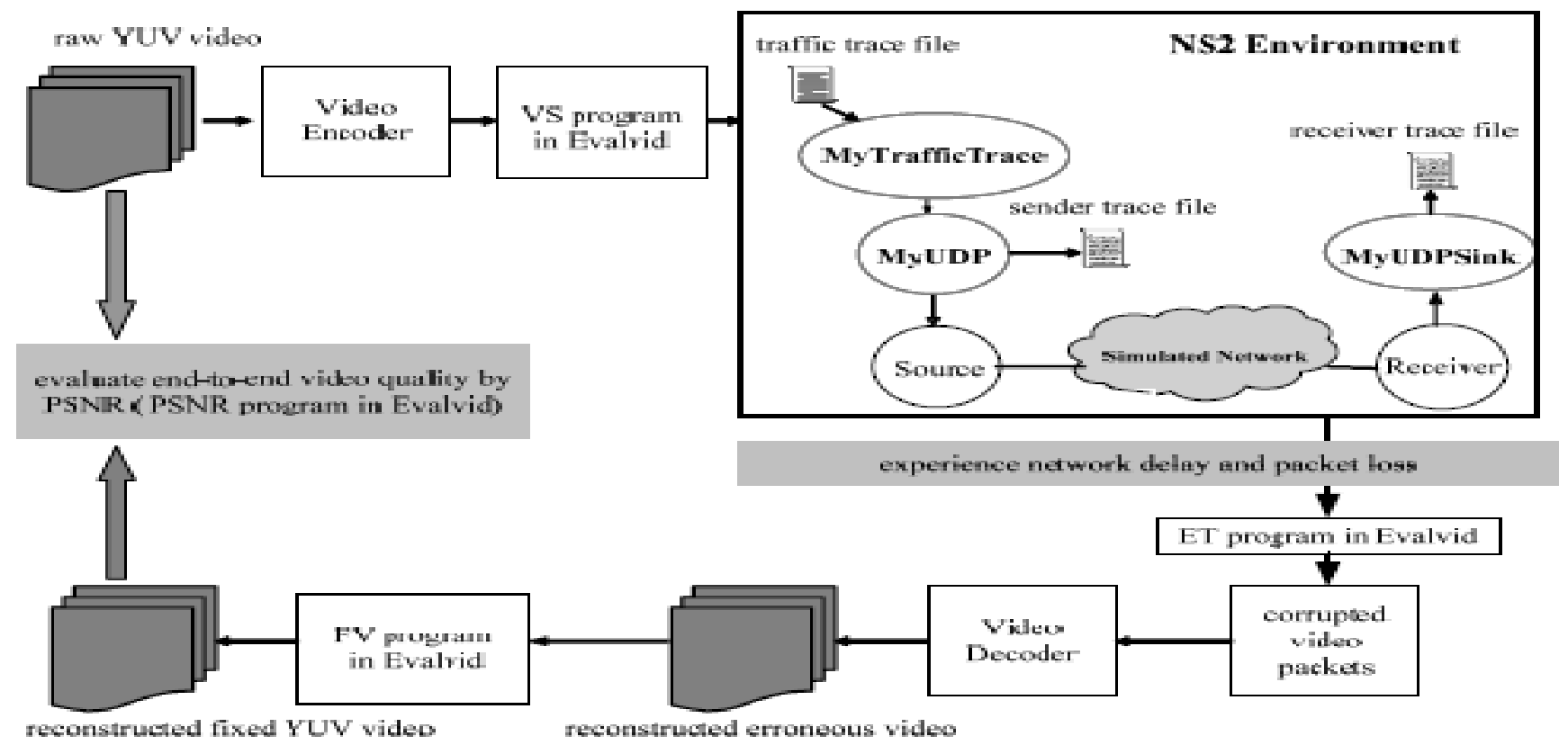

Fig 1: EvalVid Tool-set

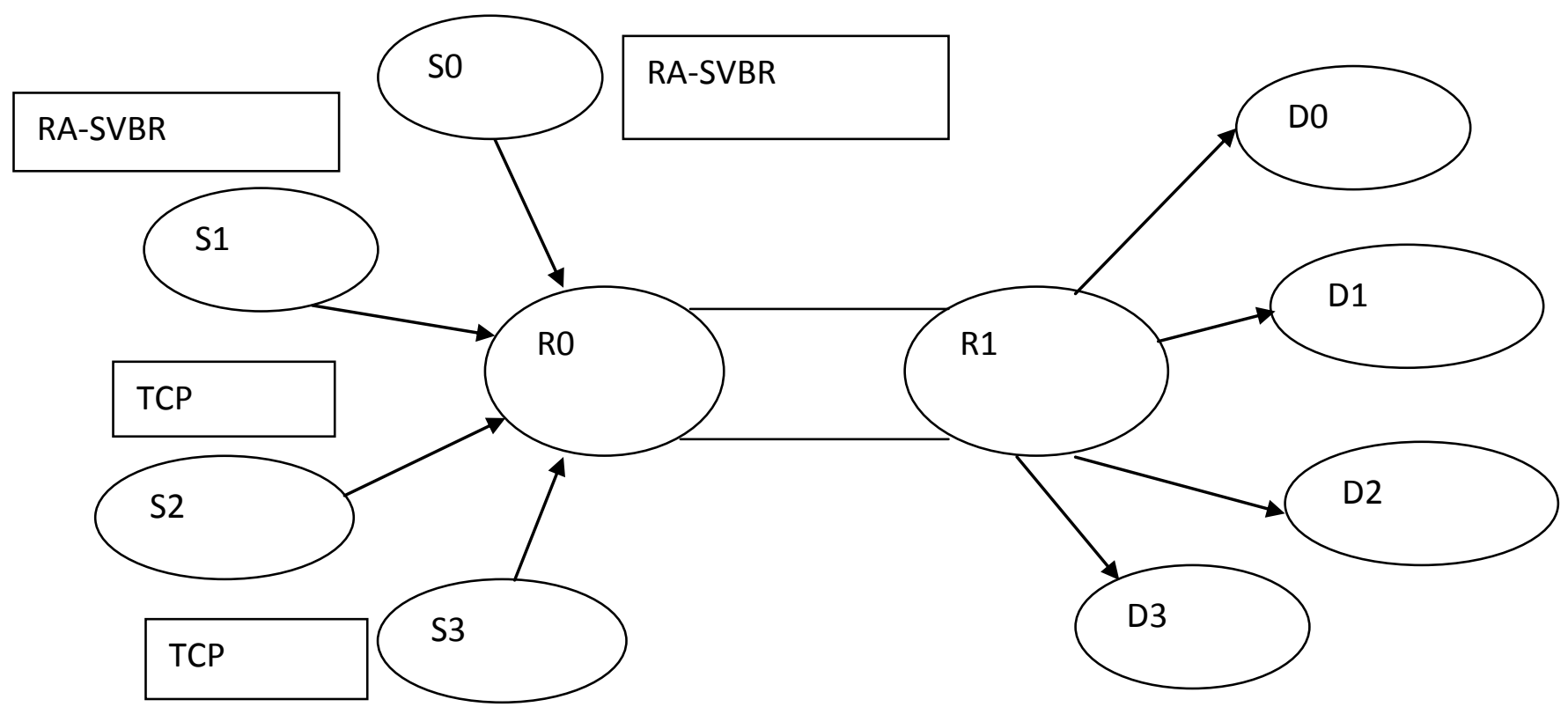

Fig 2: Simulation Topology 


\subsection{Simulation Network Topology}

Our simulation topology in this paper is the same simple topology used in [22]. As shown in Fig. 2, it is a simple dumbbell topology composed of four traffic sources and four traffic destinations. We used ns-2.30 as our network simulator to build this topology. Both $\mathrm{S} 0$ and $\mathrm{S} 1$ are fed with the simulated video trace files while S2 and S3 generate TCP traffic. The video traffic is shaped variable bit-rate traffic rate adaptive (RASVBR), and the bottleneck between the routers R0 and R1 is $40 \mathrm{Mbit} / \mathrm{s}$ link with propagation delay of $10 \mathrm{~ms}$. The access network capacities were set to $32 \mathrm{Mbit} / \mathrm{s}$ with $5 \mathrm{~ms}$ delay producing a one-way propagation delay of $20 \mathrm{~ms}$. The fair share after starting all sources was over $625 \mathrm{Kbit} / \mathrm{s}$ and both R0 and R1 routers used ordinary random early detection (RED).

Using ns-2.30 allowed for testing TFRC with different $\mathrm{Nfb}$ values aiming at reaching its optimum value required for ETFRC which enables for the best PSNR values.

\subsection{Video Sequences Used in Simulations}

Twelve video sequences were used in our simulations as shown in Table 1. They are provided for research purposes by Arizona State University research group in [24]. They were meant to vary in length, content type, and motion complexity scale. Their frames sizes were common intermediate format CIF with the size of $352 \times 288$ pixels. Their number of frames ranged from 90 to 2101 frames and their content type covered the categories of news, sports, monitoring hall videos, and others. Their motion complexity scale specified ranged from low-motion to mediummotion and high-motion.

$$
\begin{aligned}
& \operatorname{PSNR}(d B)=20 \log _{10} \frac{V_{\text {peak }}}{M S E} \\
& V_{\text {peak }}=2^{k}-1
\end{aligned}
$$

Where $k$ and MSE are as follows:

- $\quad k$ : the number of bits per pixel

- MSE: the mean square error of the luminance component.

This group of video clips is an expressive sample of video sequences streamed over Internet. They were used in YUV format where they are firstly encoded using ffmpeg.exe and then passed by the mp4.exe which are both component files of the used tool-set.

A brief description for each of the videos content is presented in the following lines:

1. Akiyo: a news reporter talking

2. Bridge-close: a close scene of Charles bridge

3. Container: a container ship moving slowly

4. Flower: a flower scene

5. Football: a high-motion football game

6. Mobile: panning of moving toys
7. Mother-daughter: Mom and daughter speaking to camera

8. News: two news reports talking

9. Silent: a person demonstrating sign language

10. Stefan: a tennis player

11. Tempete: a moving camera

12. Waterfall: a waterfall natural scene

The final output of the tool-set is a text file that contains a table of two columns where the PSNR value of each compared video frame calculated according to equation (2) is recorded in decibels $(\mathrm{dB})$ corresponding to their frame numbers. We utilized the mean PSNR value for each simulated video file for our evaluation purpose to be compared with the reference mean PSNR produced by TFRC having the default $\mathrm{Nfb}$ value of one.

The following table shows the video sequences used and their number of frames and complexity of motion:

\begin{tabular}{|c|c|c|c|}
\hline$\#$ & Video & $\begin{array}{c}\text { \# of } \\
\text { Frames }\end{array}$ & $\begin{array}{c}\text { Motion } \\
\text { Complexity }\end{array}$ \\
\hline 1 & Akiyo & 300 & Low \\
\hline 2 & Bridge-close & 2001 & Low \\
\hline 3 & Container & 300 & Low \\
\hline 4 & Flower & 250 & Low \\
\hline 5 & Football & 130 & High \\
\hline 6 & Mobile & 300 & Medium \\
\hline 7 & Mom-daughter & 300 & Low \\
\hline 8 & News & 300 & Low \\
\hline 9 & Silent & 300 & Low \\
\hline 10 & Stefan & 90 & High \\
\hline 11 & Tempete & 260 & Low \\
\hline 12 & Waterfall & 260 & Low \\
\hline
\end{tabular}

The resulting text files are then fed into the ns-2 simulation file where the output is concatenated through et_ra.exe tool and then decoded. The decoded file quality is the compared to the original file quality using the psnr.exe program as demonstrated in Fig. 1.

\section{SIMULATION RESULTS}

The goal of building and running the simulated environment explained above was to investigate the effect of switching the $\mathrm{Nfb}$ parameter over a range of values on the resulting PSNR values of the output files.

Our results are base on calculating the mean PSNR values for each video sequence which are the results of comparing the output video files of simulation over TFRC protocol and the original video files.

Those PSNR values were calculated using the psnr.exe program 
as a part of the EvalVid tool-set. The program compares each output video file to the original file on frame-by-frame basis. It produces a text file that contains a PSNR value for each of the video frames. The mean of those PSNR values were computed to be compared to those of the default PSNR values of the TFRC

Knowing that the default value of $\mathrm{Nfb}$ in TFRC is " 1 ", we managed to switch $\mathrm{Nfb}$ over the values of $1,2,4,6$, and 8 where the value of " 1 " was used as reference for testing the increase or decrease in PSNR for the other values of $\mathrm{Nfb}$.

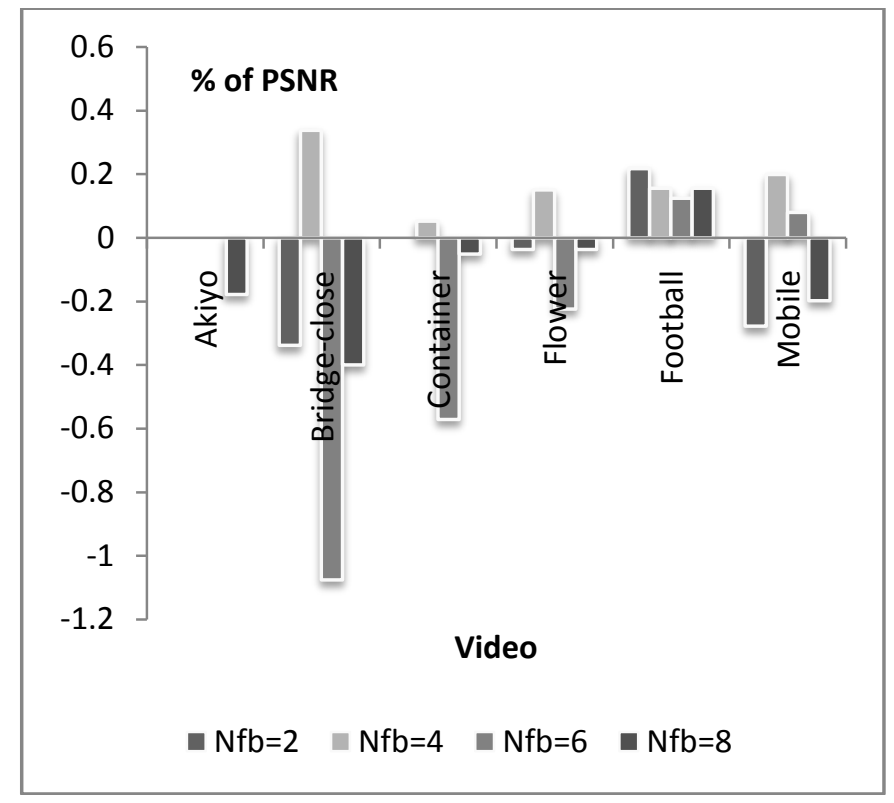

Fig. 3 Percentage of PSNR Values I

Our first finding was that the PSNR values of the output files all lie in the acceptable range at the chosen values of $\mathrm{Nfb}$. This means that TFRC is a suitable candidate for running the media traffic from both points of views of TCP-friendliness and media quality maintenance. The output files were visually meaningful. They had a degraded quality with respect to the original video files, but all the files were considered to be visually acceptable by human eye as well as PSNR values.

Our next goal in this paper was specifying the exact value of $\mathrm{Nfb}$ that produces the maximum PSNR for the output video files, and that would be set as the $\mathrm{Nfb}$ in the suggested ETFRC. To achieve this goal we measured the percentage of increase in PSNR for each of the values chosen for $\mathrm{Nfb}$ relative to the PSNR value reached by the default Nfb value of one. Fig. 3 and Fig. 4 represent the percentage of increase in PSNR achieved at $\mathrm{Nfb}=2,4,6$, and 8 relative to that achieved at $\mathrm{Nfb}=1$. Fig. 3 represents the results of the first six video sequences used while Fig. 4 represents those of the other six videos where the percentages are calculated according to equation (3).

$$
\% \text { of } P S N R=\frac{(P S N R \mid N f b=n)-(P S N R \mid N f b=1)}{(P S N R \mid N f b=1)} \times 100
$$

It is clear from the graphs that $\mathrm{Nfb}=4$ is the only value for $\mathrm{Nfb}$ that recorded positive values for the percentage of increase in PSNR for all of the twelve used video sequences. Some negative values were recorded with each of the $\mathrm{Nfb}=2,6$, and 8 which means that those values may lead to decrease in PSNR in contrary to our goal. We can also note that $\mathrm{Nfb}=4$ leads to the maximum values among the other tested values.

The average value of increase for all the twelve videos at $\mathrm{Nfb}=4$ is $0.2 \%$ which is a good increase in decibels and it also means that our proposed ETFRC can record an increase of $0.2 \%$ in PSNR values over TFRC. Other tested $\mathrm{Nfb}$ values recorded negative averages of $-0.003,-0.21$, and -0.22 for $\mathrm{Nfb}=2,6$ and 8 respectively. This means that using such values for $\mathrm{Nfb}$ may lead to decreasing the PSNR values of the video traffic, and consequently we did not select any of them for ETFRC.

We recall that the videos used for these simulations represent an expressing sample for the streamed video clips on the net either in real-time or on demand. This makes our simulation results very helpful for measuring the performance of the TFRC and enables the simulations results to resemble those of the realworld.

These simulations made all files of the same frame size pass by the same encoding and simulation circumstances so that the only factor that will let their mean PSNR values differ would be their differences in content and motion scale.

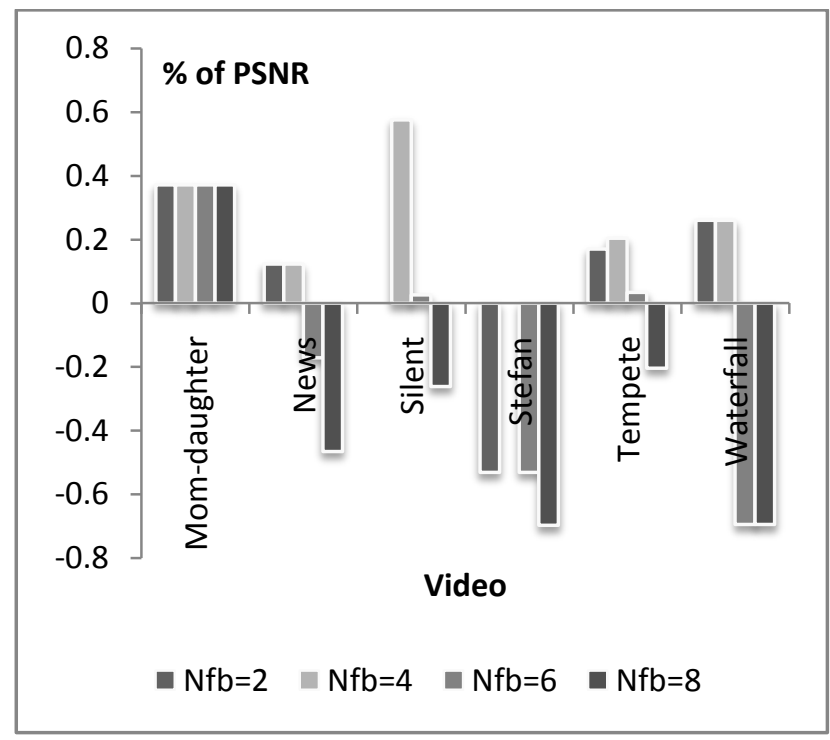

Fig. 4 Percentage of PSNR Values II

As shown in Fig. 2, it is a simple dumbbell topology composed of four traffic sources and four traffic destinations. We used ns2.30 as our network simulator to build this topology.

Both S0 and S1 are fed with the simulated video trace files while S2 and S3 generate TCP traffic. The video traffic is shaped variable bit-rate traffic rate adaptive (RA-SVBR), and the bottleneck between the routers R0 and R1 is $40 \mathrm{Mbit} / \mathrm{s}$ link with propagation delay of $10 \mathrm{~ms}$. The access network capacities were set to $32 \mathrm{Mbit} / \mathrm{s}$ with $5 \mathrm{~ms}$ delay producing a one-way propagation delay of $20 \mathrm{~ms}$. The fair share after starting all 
sources was over $625 \mathrm{Kbit} / \mathrm{s}$ and both $\mathrm{R} 0$ and $\mathrm{R} 1$ routers used ordinary random early detection (RED).

\section{CONCLUSION}

Using ns-2.30 allowed for testing TFRC with different $\mathrm{Nfb}$ values aiming at reaching its optimum value required for ETFRC which enables for the best PSNR values.

The problem of Internet congestion control has been handled by researchers over the last decade. It was observed that the quantity of media traffic traversing the Internet has tremendously increased due to the increase in the number of emerging applications running such traffic which led to worsening the case of congestion.

Several congestion control protocols have been developed to face the problem and also have been tested from the TCPfriendliness point of view and achieved promising performance in many cases, but the media traffic currently booming over Internet imposed some additional criteria on the congestion control protocols other than just being TCP-friendly and fair in bandwidth acquiring. These criteria focused on delivering the media traffic with an acceptable PSNR quality leading to a visually meaningful and acceptable traffic.

TFRC according to researches is a good candidate protocol for this target. It can balance between accomplishing the TCPfriendliness task and allowing for some QoS constraints to be met. Several researches pointed out that TFRC with its current mechanism of work is not the ideal protocol for congestion handling, and hence many enhancement attempts were made to reach a more suitable form of it. A number of research work targeted the evaluation of TFRC in terms of TCP-friendliness and fairness and many tests were done for this purpose either in the simulated environments or in the real-world. TFRC has also been tested so far regarding the quality of the media traffic running over it.

This work managed to find an enhanced version of TFRC named as ETFRC through changing the default parameter of $\mathrm{Nfb}$ in TFRC from one to be four in ETFRC. This was by testing the quality of an expressive sample group of videos when transmitted over TFRC while switching the value of $\mathrm{Nfb}$ over a range of nominated integers. This testing utilized the simulation environment and was made in terms of the visual usefulness of the received video files. It was also made in terms of the mean PSNR values in $\mathrm{dB}$ of the output files of the simulation process when compared to the originally transmitted files over TFRC. Simulations used TFRC with different $\mathrm{Nfb}$ values as the transport protocol for the media files and meant to compare the quality of the video files after being transmitted over TFRC with their quality before transmission.

TFRC was shown to produce acceptable quality for the received video files. This emphasizes the fact that TFRC is still the candidate for the congestion control problem solving. It was also shown through the simulations results that the performance of TFRC in terms of quality was enhanced slightly with the increase in the feedback frequency from one feedback per RTT to four. This enhancement is expressed as an increase in the produced PSNR values for the transmitted media traffic compared to those recorded with the default TFRC. Other tested values for the feedback frequency recorded a decrease in the PSNR values of the produced videos compared to the same reference.

We hereby propose the ETFRC protocol as an enhancement for TFRC where the feedback frequency utilized value is four instead of one. We also believe that this work can be helpful for researchers handling the congestion control problem and researchers trying to enhance the performance of TFRC in order to increase its capabilities of being the congestion control problem solution.

\section{REFERENCES}

[1] Widmer, R. Denda, and M. Mauve, "A survey on tcpfriendly congestion control (extended version)," Technical Report, Department for Mathematics and Computer Science, University of Mannheim, Feb. 2001.

[2] S. Floyd, M. Handley, J. Padhye, and J. Widmer, "Equation-based congestion control for unicast applications," in Proc. ACM SIGCOMM, Stockholm, Sweden, Aug. 2000, pp. $43-56$.

[3] S. Tsao, Y. Lai, and Y. Lin, "Taxonomy and Evaluation of TCP-Friendly Congestion Control Schemes on Fairness, Aggressiveness, and Responsiveness." IEEE Network, vol. 21 , no. 6,2007 , pp. 6-15.

[4] Lisong $\mathrm{Xu}$ and Josh Helzer, "Media Streaming via TFRC: an Analytical Study of the Impact of TFRC on Userperceived Media Quality," Computer Networks, vol. 51, no. 17, 2007, pp. 4744-4764.

[5] M. A. Talaat, M. A. Koutb, and H. S. Sorour, "PSNR Evaluation for Media Traffic over TFRC," International Journal of Computer Networks and Communications, vol. 1, no. 3, pp. 71-76, October 2009.

[6] R. Rejaie, M. Handley, and D. Estrin, "Rap: An end-to-end rate-based congestion control mechanism for real-time streams in the internet," in Proc. IEEE Infocom, Mar. 1999.

[7] D. Sisalem and A. Wolisz, "Lda+ tcp-friendly adaptation: A measurement and comparison study," in Proc. International Workshop on Network and Operating Systems Support for Digital Audio and Video (NOSSDAV), June 2000.

[8] I. Rhee, N. Balaguru, and G. Rouskas, "Mtcp: scalable tcplike congestion control for reliable multicast," in Proc. of IEEE INFOCOM, March 1999, vol.3, pp. 1265-1273.

[9] J. Padhye, D. Kurose, and R. Towsley, "A model based tcpfriendly rate control protocol," in Proc. International Workshop on Network and Operating Systems Support for Digital Audio and Video (NOSSDAV), June 1999.

[10] B. Zhou, C. P. Fu, V. O. K. Li, "Tfre veno: an enhancement of TCP-friendly rate control over wired/wireless networks,"in ProcIEEE ICNP, Oct. 2007.

[11] O. Mehani and R. Boreli, "Adapting TFRC to mobile networks with frequent disconnections," in Proc. CoNEXT 2008, 4th ACM International Conference on emerging 
Networking EXperiments and Technologies, K. W. Ross and L. Tassiulas, Eds. New York, NY, USA: ACM, 2008.

[12] D. Darjanovic and Michael Welzl, "Multfrc: providing weighted fairness for multimedia applications (and others too!)." in Proc. ACM Computer Communication Review, 2009.

[13] E. Tan, J. Chen, S. Ardon, and E. Lochin, "Video tfrc," IEEE International Conference on Communications, Beijing, China, 2008.

[14] A. Huszak and S. Imre, "Tfrc-based selective retransmission for multimedia applications," in Proc. Advances in Mobile Multimedia, Jakarta, Indonesia, 2007, pp. 53-64.

[15] M. Li, C. Lee, E. Agu, M. Claypool, and R. Kinicki, "Performance enhancement of TFRC in wireless ad-hoc networks," in Proc of. 10th International Conference on Distributed Multimedia Systems (DMS), California, USA, September 2004.

[16] G. Jourjon, E. Lochin, L. Dairaine, P. Senac, T. Moors, and A. Seneviratne, "Implementation and performance analysis of a QoS-aware TFRC mechanism," in Proc. of IEEE ICON, Singapore, September 2006.

[17] P. G. Vasallo, "Variable packet size equation-based congestion control," International Computer Science Institute ICSI, Technical Report, April, 2000.

[18] Zhuonong Xu, Jiawei Huang, and Jianxin Wang, Jin Ye, "An enhanced tfrc protocol based on step ecn marking,"
International Conference on Communications and Mobile Computing (CMC), Shenzen, China, April 2010.

[19] Dino M. Lopez-Pacheco, Emmanuel Lochin, Golam Sarwar, and Roksana Boreli, "Understanding the impact of tfrc feedbacks frequency over long delay links," Global Information Infrastructure Symposium (IEEE GIIS 2009), Hammamet, Tunisia, 2009.

[20] University of California Berkeley, "The Network Simulator - ns-2," [Online] http://www.isi.edu/nsnam/ns/.

[21] C. H. Ke, C. K. Shieh, W. S. Hwang, A. Ziviani, “An Evaluation Framework for More Realistic Simulations of MPEG Video Transmission", Journal of Information Science and Engineering, vol. 24, no. 2, pp.425-440, March 2008

[22] J. Klaue, B. Rathke, and A. Wolisz, "Evalvid - A framework for video transmission and quality evaluation," in Proc. 13th International Conference on Modeling Techniques and Tools for Computer Performance Evaluation, pp. 255-272, Urbana, Illinois, USA, September 2003

[23] A. Lie, and J. Klaue "Evalvid-RA: Trace Driven Simulation of Rate Adaptive MPEG-4 VBR Video", Multimedia Systems, vol. 14, no. 1, 13. November 2007.

[24] Arizona State University, "YUV Video Sequences" [Online] http://trace.eas.asu.edu/yuv/index.html 